\title{
Characterization of porous low- $k$ films using variable angle spectroscopic ellipsometry
}

\author{
M. T. Othman, J. A. Lubguban, A. A. Lubguban, and S. Gangopadhyay ${ }^{a}$ \\ Department of Electrical and Computer Engineering, University of Missouri, Columbia, Missouri 65211 \\ R. D. Miller, W. Volksen, and H.-C. Kim \\ IBM Almaden Research Center, San Jose, California 95120
}

(Received 8 August 2005; accepted 28 February 2006; published online 19 April 2006)

\begin{abstract}
Variable angle spectroscopic ellipsometry (VASETM) is used as a tool to characterize properties such as optical constant, thickness, refractive index depth profile, and pore volume fraction of single and bilayer porous low- $k$ films. The porous films were prepared using sacrificial pore generator (porogen) approach. Two sets of porous films with open- and closed-pore geometries were measured. Three models were used for data analysis: Cauchy, Bruggeman effective medium approximation (BEMA), and graded layer. Cauchy, a well-known model for transparent films, was used to obtain thickness and optical constant, whereas BEMA was utilized to calculate the pore volume fraction from the ellipsometric data. The Cauchy or BEMA models were then modified as graded layers, resulting in a better fit and a better understanding of the porous film. The depth profile of the porous film implied a more porous layer at the substrate-film interface. We found 3\%-4\% more porosity at the interface compared with the bulk for both films. This work shows that VASE ${ }^{\mathrm{TM}}$, a nondestructive measurement tool, can be used to characterize single- and multigraded layer porous films quickly and effectively. (C) 2006 American Institute of Physics. [DOI: 10.1063/1.2189018]
\end{abstract}

\section{INTRODUCTION}

Porous thin films are promising candidates in a variety of applications such as antireflection coatings and waveguides in optoelectronics and as intermetal dielectrics (IMD) in microelectronics. ${ }^{1,2}$ In ultra-large-scale integration (ULSI) devices, the International Technology Roadmap for Semiconductors (ITRSs) predicted that by year 2010, a $45 \mathrm{~nm}$ technology with 12 metal levels will be used. One of the requirements to achieve this technology is to find an IMD with an effective dielectric constant $(k)$ of less than 2.1. ${ }^{3}$ Incorporating porosity in insulator layers can reduce $k$ to desired values. ${ }^{4}$ The pore size should be carefully controlled and should be smaller than the minimum features size to achieve mechanical stability. ${ }^{5}$ It is also preferable to have a homogenous pore size distribution to prevent $k$ anisotropy. Thus, characterizations of porous films are becoming crucial and increasingly difficult. Conventional measurement techniques have become unreliable because of very small pore sizes in the order of few nanometers.

Well-known methods to determine the pore size distribution, pore volume fraction, and whether it is an open- or closed-pore structure are based in different physicochemical principles. ${ }^{1}$ The available techniques can be divided into three main categories: (1) scattering techniques-small-angle $\mathrm{x}$-ray spectrometry (SAXS) and x-ray reflectivity (XRR); (2) adsorption techniques-ellipsometric porosimetry (EP); (3) positron annihilation lifetime spectroscopy (PALS). SAXS is based on diffuse scattering of $\mathrm{x}$ rays from heterogeneous electron density distributions. This scattering technique is

\footnotetext{
a) Author to whom correspondence should be addressed; electronic mail: gangopadhyays@missouri.edu
}

normally used in combination with XRR that gives the information of total porosity. ${ }^{6,7}$ Adsorption porosimetry is a widely used method in characterizing pore structure. ${ }^{1,8} \mathrm{EP}$ is a combination of nonintrusive (wave propagation) and intrusive (adsorption, gas) methods. The main goal of EP is to measure the optical constant of a porous material when the pores adsorp/desorp vapor with known optical constant. ${ }^{1,9,10}$ PALS is based on positronium lifetime during collisions with the pore surfaces. PALS has the capability to determine the pore size distribution and to distinguish pore structure (open or closed). ${ }^{11}$ The techniques summarized above may be time consuming, requiring difficult sample preparation, and may render the sample unusable after measurements.

In this paper, we report variable angle spectroscopic ellipsometry (VASETM, J.A. Wollam, Inc.) as a tool to investigate properties of porous films such as pore volume fraction and refractive index depth profile in addition to thickness and refractive index usually obtained by ellipsometry. The refractive index profile is suggestive of the distribution of pores in the films. Spectroscopy ellipsometry also provides an accurate measurement of ellipsometric data which are required for adequate characterization of unknown sample. ${ }^{12,13}$ With an accurate modeling, we can determine the percentage porosity at the interface and bulk porous. We can also distinguish either a porous sample has a graded or nongraded refractive index throughout sample thickness.

Spectroscopic ellipsometry is a quick, noncontact, and nondestructive optical technique for thin film characterization based on the polarization change occurring when light interacts with materials. ${ }^{12,13}$ Spectroscopic ellipsometry measures $\Psi$ and $\Delta$ values as a function of wavelength and incident angle. ${ }^{14}$ These numbers are related to the complex Fresnel coefficient for $p$ and $s$ polarized light. 


$$
\rho=\frac{R_{p}}{R_{s}}=\tan (\Psi) e^{i \Delta},
$$

where $R_{p}$ and $R_{s}$ are the total reflection coefficients for a plane wave polarized light parallel $(p)$ and perpendicular $(s)$ to the plane of incidence, respectively. ${ }^{15,16}$ The mathematical expressions of $R_{p}$ and $R_{s}$ are derived from Maxwell's equations for electromagnetic radiation applied to the boundary between dissimilar materials related to the Fresnel reflection coefficient and layer thickness, hence, interrelated with optical constant of materials. ${ }^{17-19}$

Ellipsometry does not directly measure the above properties such as refractive index or pore volume fraction but it measures $\Psi$ and $\Delta$. To extract these parameters, it is necessary to perform a model dependent analysis of the measured $\Psi$ and $\Delta .{ }^{20}$ After data are acquired over the desired spectral range at different incident angles, models for the optical structure of the substrate and sample are constructed. Then, the Fresnel equations based on the model are used to predict the expected $\Psi$ and $\Delta$ values. The measured $\Psi$ and $\Delta$ values are compared with the predicted values. The $\Psi$ and $\Delta$ are functions of optical constant and thickness which can also be the adjustable model parameters. The analysis procedure is called data fitting, because the model parameters are varied to find the best fit between generated and measured data. The most common fit parameters are optical constant and thicknesses. The fitting algorithm used in VASETM is the Marquardt-Levenberg algorithm. The root mean square error (MSE) is used to quantify the difference between the experimental and predicted data.

This paper will show analysis of two types of porous film, i.e., those with open-pore and closed-pore geometries, respectively. The open-pore film has a low density, a low refractive index of 1.12, and has a low mechanical strength that may not be practical for use as a dielectric in ULSI devices. However, the closed-pore film has a higher density and higher mechanical strength. We will also analyze porous films that are graded (refractive index varies with across the film thickness) with a single or bilayer structure, nongraded single layer and also porous film treated with oxygen plasma to damage the film surface. Although some films here cannot survive chemical mechanical polishing in ULSI processing, the analysis of these films shows the benefits of using a VASETM method for porous films used not only in microelectronic applications but others as well.

\section{EXPERIMENT}

Single and bilayer porous samples were used for VASETM measurements. A sacrificial-porogen technique based on the selective removal of the organic macromolecular porogen from phase-separated mixtures of organic/ inorganic nanohybrids used in this experiment was prepared and supplied by International Business Machines Corp. (IBM ${ }^{\mathrm{TM}}$ ) Almaden Research Center. ${ }^{21}$ Two sets of these porous films with open- or closed-pore geometries were used. These films were fabricated by mixing appropriate ratios of porogen and matrix precursor; $25 \%$ by weight porogen loading for closed pore and 55\% by weight porogen loading for open pore, respectively. The sacrificial porogen is of linear poly(propylene)glycol (PPG) with molecular weight MW $=20000 \mathrm{~g} / \mathrm{mol}$, and the matrix precursor is of poly(methyl silsesquioxane) (PMSSQ). The solvent used for both PPG and PMSSQ solutions was propylene glycol methyl ether acetate (PGMEA). The mixture of PPG and PMSSQ solution was spun on silicon substrates and was heated briefly to $50{ }^{\circ} \mathrm{C}$ for solvent removal before curing at $200{ }^{\circ} \mathrm{C}$ in nitrogen to produce phase-separated structure. The samples were then annealed at $450{ }^{\circ} \mathrm{C}$ in $\mathrm{Ar}$ for $2 \mathrm{~h}$ at $5{ }^{\circ} \mathrm{C} / \mathrm{min}$ ramping to remove the PPG and create porosity in the films. Further details of the sample preparation and results can be found in a previous paper. ${ }^{22}$ Transmission electron micrographs of the samples were also taken previously to determine the pore morphology. ${ }^{22}$ The verification of the porous structure of the above films has been described thoroughly in these previous experiments. ${ }^{22}$ Based on these measurements, the open-pore sample has $67.1 \%$ porosity and pore size $(2-20 \AA)$, whereas the closed-pore film has $27 \%$ porosity and pore size $(2-8 \AA)$. These results will be compared to our results using spectroscopy ellipsometry. The bilayer film is generated by mixing different wt $\%$ solution of a commercially available organosilicates precursor. The top layer has $70 \%$ porogen loading and the bottom layer has $40 \%$ porogen loading. The details of bilayer sample preparation can be found elsewhere. ${ }^{21}$

We also prepared another set of porous films by mixing $50 \%$ PPG (MW $=600 \mathrm{~g} / \mathrm{mol})$ and 50\% PMSSQ. PGMEA was used as solvent for both PPG and PMSSQ solutions. We spin coated the precursor and directly baked in air for $3 \mathrm{~min}$ at $200{ }^{\circ} \mathrm{C}$ to thermally decompose the porogen.

In order to obtain the pore volume fraction using Bruggeman effective medium approximation (BEMA), a set of dense PMSSQ optical constants are needed. This nonporous PMSSQ film was prepared by spin coating a solution without the porogen. $\mathrm{A} \mathrm{SiO}_{2}$ film is also prepared for comparison with the porous film. This $\mathrm{SiO}_{2}$ was processed by wet oxidation for $40 \mathrm{~min}, 3.5 \mathrm{SCCM}$ (SCCM denotes cubic centimeter per minute at STP) $\mathrm{O}_{2}$ flow rate at a temperature of $1100{ }^{\circ} \mathrm{C}$.

We also studied porous organosilicate film treated with oxygen plasma. This is to simulate a film which has a surface property different from the bulk, which is because of $\mathrm{O}_{2}$ plasma damage in the surface. The closed-pore sample was exposed to oxygen plasma via reactive ion etching with $20 \mathrm{SCCM}$ of $\mathrm{CF}_{4}$ and $80 \mathrm{SCCM}$ of $\mathrm{O}_{2}$ for $1 \mathrm{~min}$ at $200 \mathrm{~W}$ plasma power. ${ }^{23}$

For ellipsometric data modeling, Cauchy, BEMA, or graded layer are used for the porous film. For the silicon substrate, we have chosen silicon optical constants obtained by Herzinger et al. which cover a broader range $(188-6200 \mathrm{~nm}) .{ }^{24}$ Similar optical constants obtained by Jellison and Aspnes, available from Palik's handbook, can also be used. ${ }^{25}$ The choice of which depends on the wavelength range. The Cauchy dispersion relation has been used to model transparent materials, such that the refractive index $n$ decreased with increasing wavelength. The Cauchy equation can be expressed approximately as a refractive index $n$ as a function of wavelength $\lambda,{ }^{12,13}$ 


$$
n(\lambda)=n_{0}+\frac{n_{1}}{\lambda^{2}}+\frac{n_{2}}{\lambda^{4}}
$$

where $n_{0}, n_{1}$, and $n_{2}$ are known as Cauchy coefficients. $n_{0}$ is the constant that dominates $n(\lambda)$ for long wavelengths, $n_{1}$ controls the curvature of $n(\lambda)$ in the middle of the visible spectrum, and $n_{2}$ influences $n(\lambda)$ to a greater extent in shorter wavelengths. The parameters, $n_{0}, n_{1}$, and $n_{2}$, and the thickness of the film were used to calculate $\Psi$ and $\Delta$ values. Refractive index and thickness were used to calculate the Fresnel reflection coefficients and the total reflection coefficient which were related to the measured $\Psi$ and $\Delta$ values. The calculated $\Psi$ and $\Delta$ values were compared to measured $\Psi$ and $\Delta$ values.

BEMA is a model that is based on the intermixed composites made up of uniform cells, which all together fit the space completely. ${ }^{26}$ The BEMA layer provides a method to combine two sets of optical constants together and the percentage of the mixture. The optical constants used for the porous film studied here are dense PMSSQ $\left(n_{\mathrm{PMSSQ}}=1.39\right)$ and air $\left(n_{\text {air }}=1.0\right)$. The Bruggeman expression is given as ${ }^{27}$

$$
f_{a} \frac{\varepsilon_{a}-\varepsilon}{\varepsilon_{a}+2 \varepsilon}+f_{b} \frac{\varepsilon_{b}-\varepsilon}{\varepsilon_{b}+2 \varepsilon}=0
$$

where $\varepsilon$ is the effective dielectric function of the composite material. $\varepsilon_{a}$ and $\varepsilon_{b}$ are the dielectric functions of phases $a$ and $b$ in their pure forms. $f_{(a \text { or } b)}=n_{(a \text { or } b)} /\left(n_{a}+n_{b}\right)$ is the volume fractions of the materials $a$ and $b$. The optical constants can be presented both in the form of complex refractive index $(N=\sqrt{\varepsilon}=n+i k)$ and complex dielectric form $(\varepsilon$ $\left.=\varepsilon_{1}+i \varepsilon_{2}\right)$. ${ }^{1,18,25}$ The graded model works by creating a series of thin homogenous layers with optical constants that slightly change in each layer from the sample surface to the substrate-sample interface.

As stated in the introduction, spectroscopic ellipsometry is a model dependent technique which compares the measured $\Psi$ and $\Delta$ data as a function of wavelength with the calculated model data. The model parameters (optical constants and sample thickness) can be varied such that the calculated data will closely match with the measured data. The merit functions that are used to judge the quality of the match between the measured and the calculated data are known as maximum likelihood estimators. The following maximum likelihood estimators or MSE are used to fit the model parameters: ${ }^{12}$

$$
\begin{aligned}
\text { MSE } & =\frac{1}{2 N-M} \sum_{i=1}^{N}\left[\left(\frac{\Psi_{i}^{\bmod }-\Psi_{i}^{\exp }}{\sigma_{\Psi, i}^{\exp }}\right)^{2}+\left(\frac{\Delta_{i}^{\bmod }-\Delta_{i}^{\bmod }}{\sigma_{\Delta, 1}^{\exp }}\right)^{2}\right] \\
& =\frac{1}{2 N-M} X^{2}
\end{aligned}
$$

where $N$ is the number of $\Psi$ and $\Delta$ data points, $M$ is the number of variable parameters in the model, and $\sigma$ is the standard deviation of the $i$ th data point. This merit function will exhibit a minimum value of zero when the calculated model matches the measured experimental data perfectly, but in practice, the model is unable to match the experimental data perfectly. ${ }^{20}$
Details and source code for implementing LevenbergMarquardt algorithm is described elsewhere. ${ }^{28}$ The important element of the fitting procedure is the curvature matrix given as

$$
\alpha_{k l}=\sum_{i=1}^{N}\left[\frac{1}{\sigma_{\Psi, i}^{2}} \frac{\partial \Psi_{i}^{\bmod }}{\partial a_{k}} \frac{\partial \Psi_{i}^{\bmod }}{\partial a_{l}}+\frac{1}{\sigma_{\Delta, i}^{2}} \frac{\partial \Delta_{i}^{\bmod }}{\partial a_{k}} \frac{\partial \Delta_{i}^{\bmod }}{\partial a_{l}}\right] .
$$

The curvature matrix is related to the covariance matrix such that $C=[\alpha]^{-1}$. Once the final fit parameters have been determined, the standard $90 \%$ confidence limit (SCL) and the figure of merit (FOM) are used to describe the confidence in the ith fit parameter given as

$$
\begin{aligned}
& \mathrm{SCL}_{i}=1.65 \sqrt{\left(C_{i i}\right)}, \\
& \mathrm{FOM}_{i}=\left(\mathrm{SCL}_{i}\right)(\mathrm{MSE}) .
\end{aligned}
$$

WVASE32®, which is an ellipsometry analysis software, generates the confidence limit FOM value after each fitting procedure. This FOM value is also known as error bar. The error bars obtained from this work are \pm 0.1 for thickness, $\pm 5 \times 10^{-5}$ for refractive index, and $\pm 1 \times 10^{-2}$ for porosity.

Ellipsometric measurements on porous PMSSQ film were taken at $65^{\circ}$ and $75^{\circ}$ incident angles at $500-1000 \mathrm{~nm}$ with $1 \mathrm{~nm}$ step interval (with adjustable autoretarder element). The primary difference between measurements with and without autoretarder element is that the ellipsometer will measure $\Delta$ between $0^{\circ}-360^{\circ}$ and $0^{\circ}-180^{\circ}$, respectively. ${ }^{12,14}$ The main advantage of not using autoretarder element is that it provides quick measurement, especially for a well-known sample, however, it will lose accuracy at or near $0^{\circ}$ or $180^{\circ}$. Thus, using the autoretarder element provides data sensitivity over the entire $0^{\circ}-360^{\circ}$ and reduce the MSE. This is important because very accurate measurements of $\Delta$ are required for adequate characterization of unknown samples. ${ }^{29}$

\section{RESULTS AND DISCUSSION}

The nonporous PMSSQ and $\mathrm{SiO}_{2}$ were analyzed first as a reference for the porous films. The Cauchy model which worked well for transparent films was used for PMSSQ films. Since PMSSQ has a O-Si-O backbone based on its empirical formula of $\left[\mathrm{CH}_{3}-\mathrm{SiO}_{3 / 2}\right]_{n}$, the film may be transparent at the above wavelengths and Cauchy model may work. Figures 1(a) and 1(b) show that the generated $\Psi$ and $\Delta$ spectra for PMSSQ are nearly indistinguishable from the experimental data. Thus, the Cauchy model works well with PMSSQ films. Excellent agreement between the experimental and generated data (not shown here) for $\mathrm{SiO}_{2}$ film were also found. The conversion of Cauchy to the graded Cauchy model did not change the $\Psi$ and $\Delta$ fit spectra. This indicates that the nonporous PMSSQ and $\mathrm{SiO}_{2}$ samples have the same refractive index across the film (i.e., nongraded) and thus have uniform density. Table I lists the thicknesses and optical constants for both PMSSQ and $\mathrm{SiO}_{2}$ films obtained from Cauchy and graded Cauchy models. The values of the optical constants from Cauchy analysis: the PMSSQ has a thickness of $701 \mathrm{~nm}$, refractive index of 1.38, and a MSE of 5.5. The conversion to a graded layer did not change the MSE significantly (4.9) resulting in similar thickness and refractive in- 

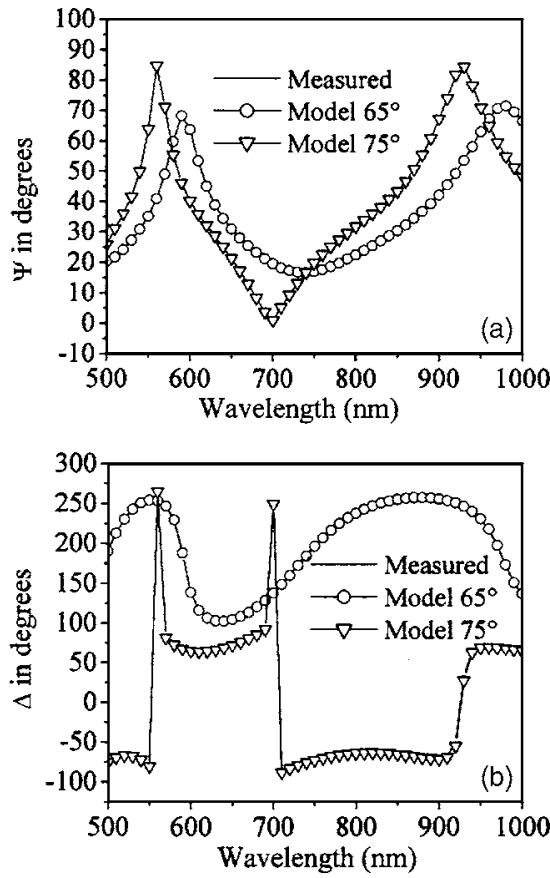

FIG. 1. Nonporous PMSSQ (a) $\Psi$ fit spectra and (b) $\Delta$ fit spectra using Cauchy model. (MSE $=5.5$ ).

dex. For $\mathrm{SiO}_{2}$ film, modifying Cauchy to graded layer does not improve the MSE either. The thickness and refractive index for $\mathrm{SiO}_{2}$ are $457 \mathrm{~nm}$ and 1.46, respectively.

Table II lists the thickness, refractive index, porosity, and MSE values obtained by fitting the open- and closed-pore PMSSQ films using Cauchy, graded Cauchy, and BEMA models. This summary of results will be referred throughout the text.

Figures 2(a) and 2(b) show the measured and generated $\Psi$ and $\Delta$ spectra for the porous PMSSQ film with open-pore structure. The Cauchy model also works well for the porous PMSSQ films. The spectra for closed-pore film were similar to open-pore film, hence, will not be shown. We obtained film thickness of $637 \mathrm{~nm}$, refractive index of 1.13, and a relatively low MSE of 3.1.

For the supposedly open-pore structure, the Cauchy model gives $637 \mathrm{~nm}$ with a low refractive index of 1.13 (Table II), implying a lower density PMSSQ and confirming the porous nature of the film (nonporous PMSSQ has refractive index of 1.38). To calculate the porosity of the film, the BEMA model was used. Two sets of optical constants are used in BEMA calculation: (i) dense PMSSQ matrix and (ii) air. The BEMA model gave a similar $\Psi$ and $\Delta$ behavior

TABLE I. List of optical constants and MSE for nonporous PMSSQ and $\mathrm{SiO}_{2}$ films modeled as Cauchy and modified graded Cauchy layers.

\begin{tabular}{llccc}
\hline \hline $\begin{array}{c}\text { Nonporous } \\
\text { sample }\end{array}$ & \multicolumn{1}{c}{ Model } & $\begin{array}{c}\text { Thickness } \\
(\mathrm{nm})\end{array}$ & $\begin{array}{c}\text { Refractive index } \\
(n)\end{array}$ & MSE \\
\hline PMSSQ & Cauchy & 701.2 & 1.388 & 5.5 \\
& Graded Cauchy & 702.3 & 1.387 & 4.9 \\
$\mathrm{SiO}_{2}$ & Cauchy & 458.7 & 1.460 & 1.8 \\
& Graded Cauchy & 457.1 & 1.463 & 4.1 \\
\hline \hline
\end{tabular}

TABLE II. List of thickness, optical constant, porosity, and MSE for openand closed-pore films using Cauchy and BEMA models with or without grading.

\begin{tabular}{|c|c|c|c|c|c|}
\hline Sample & Model & $\begin{array}{c}\text { Thickness } \\
(\mathrm{nm})\end{array}$ & $\begin{array}{l}\text { Refractive index } \\
\qquad(n)\end{array}$ & $\begin{array}{l}\text { Porosity } \\
(\%)\end{array}$ & MSE \\
\hline \multirow[t]{12}{*}{ Open pore } & Cauchy & 637.1 & 1.137 & $\cdots$ & 3.1 \\
\hline & Graded Cauchy & 638.6 & 1.137 & $\cdots$ & 1.5 \\
\hline & 1 BEMA layer & 637.1 & 1.137 & 63.3 & 3.0 \\
\hline & $\begin{array}{l}\text { Graded } 1 \\
\text { BEMA layer }\end{array}$ & 638.7 & 1.137 & 63.5 & 1.6 \\
\hline & \multicolumn{5}{|c|}{2 BEMA layers } \\
\hline & Top layer & 595.5 & 1.137 & 63.5 & 1.5 \\
\hline & Bottom layer & 43.2 & 1.125 & 66.5 & \\
\hline & Total thickness & 638.7 & & & \\
\hline & \multicolumn{5}{|c|}{$\begin{array}{c}\text { Graded } 2 \\
\text { BEMA layers }\end{array}$} \\
\hline & Top layer & 598.6 & 1.137 & 63.5 & 1.5 \\
\hline & Bottom layer & 40.2 & 1.121 & 67.5 & \\
\hline & Total thickness & 638.8 & & & \\
\hline \multirow[t]{12}{*}{ Closed pore } & Cauchy & 440.9 & 1.275 & $\cdots$ & 4.3 \\
\hline & Graded Cauchy & 440.9 & 1.274 & $\cdots$ & 2.4 \\
\hline & 1 BEMA layer & 440.7 & 1.275 & 28.6 & 4.4 \\
\hline & $\begin{array}{l}\text { Graded } 1 \\
\text { BEMA layer }\end{array}$ & 441.0 & 1.274 & 28.9 & 2.4 \\
\hline & \multicolumn{5}{|c|}{2 BEMA layers } \\
\hline & Top layer & 368.0 & 1.276 & 28.5 & 2.2 \\
\hline & Bottom layer & 72.9 & 1.261 & 32.2 & \\
\hline & Total thickness & 440.9 & & & \\
\hline & \multicolumn{5}{|c|}{$\begin{array}{c}\text { Graded } 2 \\
\text { BEMA layers }\end{array}$} \\
\hline & Top layer & 370.3 & 1.276 & 28.4 & 2.2 \\
\hline & Bottom layer & 70.4 & 1.259 & 32.7 & \\
\hline & Total thickness & 440.7 & & & \\
\hline
\end{tabular}

spectra with Cauchy model and is not shown here. This model gave $63.3 \%$ porosity with MSE value of 3.0 and thickness of $637 \mathrm{~nm}$-values are similar with those of the Cauchy model. This pore volume fraction obtained from ellipsometer analysis is lower by 3\% than previously obtained. $^{23}$ Surface roughness simulation was added on the above models, but it did not improve the fit and MSE. This also means that the porous film surface is relatively smooth $(<1 \mathrm{~nm}$ roughness). The Cauchy and BEMA model are then modified to graded layers in order to study the behavior of porous sample. The graded layer model is created by subdividing 100 thin homogenous film layers. At each layer, the optical constant will slightly change. The depth profile is analyzed at $633 \mathrm{~nm}$ wavelength. Modifying both Cauchy and BEMA models to graded models has improved the fit by $50 \%$ (i.e., MSE decreased from 3.1 to 1.5 ).

Figure 3 shows the grading profile of the refractive index at $633 \mathrm{~nm}$ from single and double BEMA layers model for open-pore sample. For open-pore film fitted with single BEMA layer model, the depth profile shows an abrupt increase in refractive index at the silicon-film interface from 1.12 to 1.135 . Then, the refractive index change very slowly 

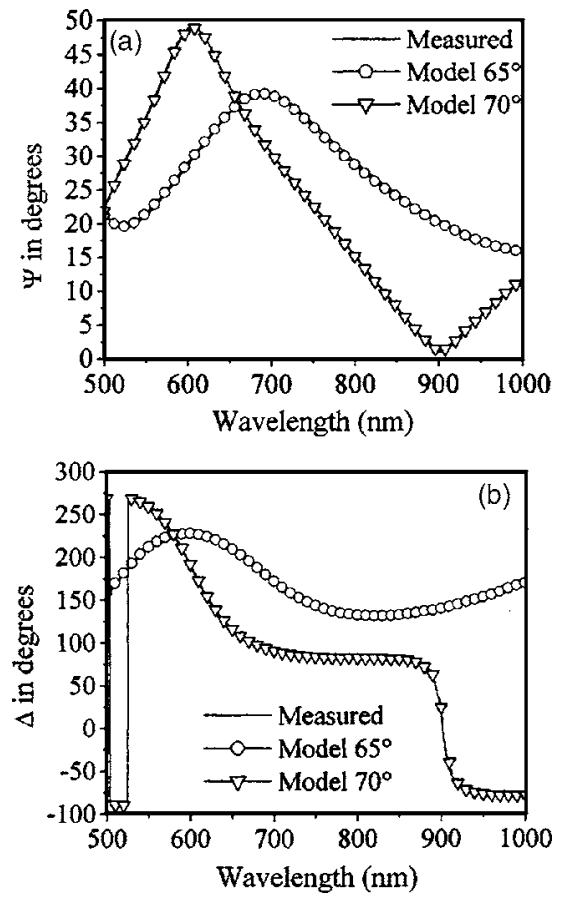

FIG. 2. Generated and experimental (a) $\Psi$ fit spectra and (b) $\Delta$ fit spectra using Cauchy model for open-pore sample with a MSE=3.1.

towards the bulk of the film and became almost constant up to the surface (1.137). Thus, the bulk film may not have the same porosity compared with the thin interface layer based on Fig. 3. The material was then modeled as two BEMA layers to find the porosity and thickness values of the thin layer at the interface and bulk, respectively. During the fitting process, the percent porosity and the thickness of the two BEMA layers are left to vary or float. The result from Table II shows that the interface layer is $43 \mathrm{~nm}$ with a porosity of $66.5 \%$, whereas the bulk has $3 \%$ less porosity (63.5\%). The MSE value for this modeling is 1.5 , which is equivalent with graded BEMA (1.5). We have also graded the two BEMA layers with the goal of lowering the MSE and have a more specific description of the film. Figure 3 shows the depth profile for open-pore structures film using two independent graded BEMA layers. The porosity at the siliconfilm interface is $67.5 \%$ and $63.5 \%$ porosity at the bulk-a difference of $4 \%$. The interface layer thickness was $40.2 \mathrm{~nm}$ while the bulk was $598 \mathrm{~nm}$. The MSE for this modeling (1.5)

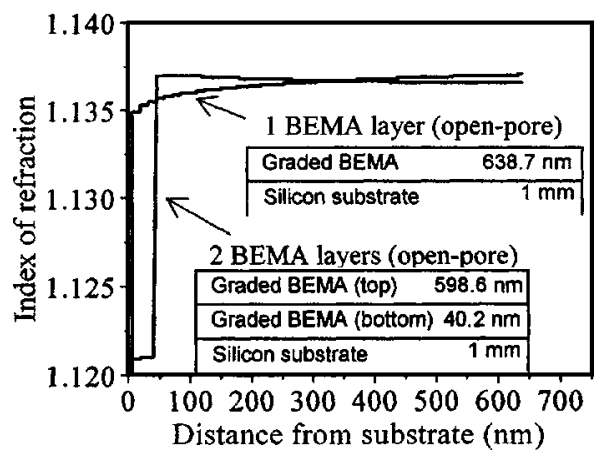

FIG. 3. Depth profiles at $633 \mathrm{~nm}$ wavelength for open-pore film using single BEMA with grading (MSE $=1.5)$ and two BEMA layers with grading $(\mathrm{MSE}=1.5)$.

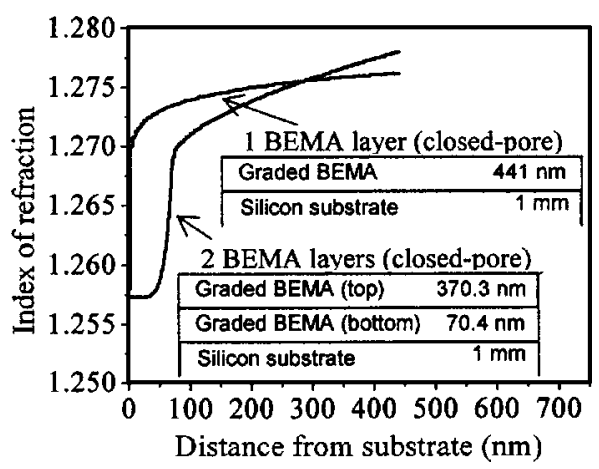

FIG. 4. Depth profiles at $633 \mathrm{~nm}$ wavelength for (1) closed-pore films using single BEMA with grading (MSE=2.2), two BEMA layers with grading (MSE $=2.2$ ), and (2) single porous film using single BEMA grading (MSE $=1.0)$.

is the same with the graded BEMA and two BEMA layer models. Since all three models gave the same MSE, either one can be used to physically describe the film. One common conclusion that can be derived in all three models is that the interface is $3 \%-4 \%$ more porous than the bulk. ${ }^{30}$ The maximum thickness of this interface layer is $43 \mathrm{~nm}$.

For the closed-pore film, similar analysis was performed as the open-pore film. The thickness of the film was $440 \mathrm{~nm}$ with a refractive index of 1.275 and MSE of 4.3 (Table II). The BEMA layer gives a porosity of $28.6 \%$ and similar thickness, refractive index, and MSE. This pore volume fraction obtained is higher by $2 \%$ from previous measurements. ${ }^{23}$ The generated and experimental $\Psi$ and $\Delta$ fit spectra show a similar characteristics with the open-pore films. We then converted the models into graded layers. Figure 4 shows the grading profile for closed-pore sample and shows the same behavior as the open-pore film. The interface has a lower refractive index and it gradually increases towards the surface of the films. The resulting MSE of the graded layers was 2.4 , a $50 \%$ improvement of the fits. The film was also modeled as two BEMA layers, but the MSE fit improvement is similar with graded model (2.2). The bottom layer $(72.9 \mathrm{~nm})$ shows a porosity of $32.2 \%$ and the bulk is $28.5 \%$. Both BEMA layers were also graded but the MSE fit remained unchanged. Figure 4 also shows depth profile for closed-pore structure using both graded BEMA layers. The bottom layer shows a thickness of $70.4 \mathrm{~nm}$ and a porosity of $32.7 \%$ while the bulk has a porosity of $28.4 \%$. Following the same arguments as the open-pore films, either the graded BEMA or Cauchy, two BEMA, and two graded BEMA models could be used to describe the closed-pore films because of similarity of MSE. However, it is clear that the substrate-film interface of the porous samples is $4 \%$ more porous than the bulk with a maximum thickness of $73 \mathrm{~nm}$. Spectroscopic ellipsometry could not distinguish between open or closedpore samples. The wavelength of the incident light $(197-2000 \mathrm{~nm})$ is far too broad to be affected with the pore sizes of the samples $(2-8 \AA){ }^{22}$ All of the models used have given almost the same total thickness.

Not all porous films are graded as we will be shown next. For directly baked porous sample (PPG MW $=600 \mathrm{~g} / \mathrm{mol}$ ), we obtained an excellent agreement between generated and experimental data by using Cauchy and 

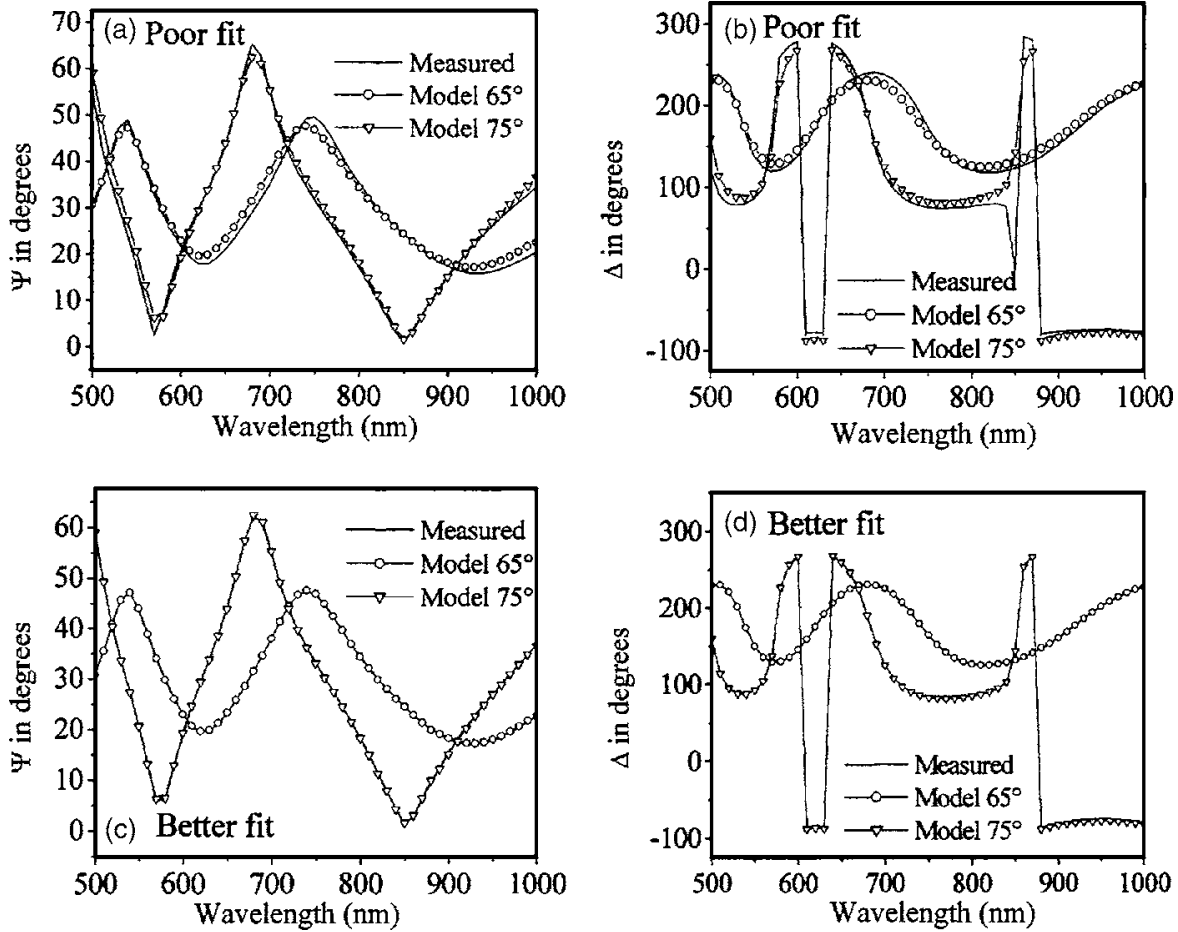

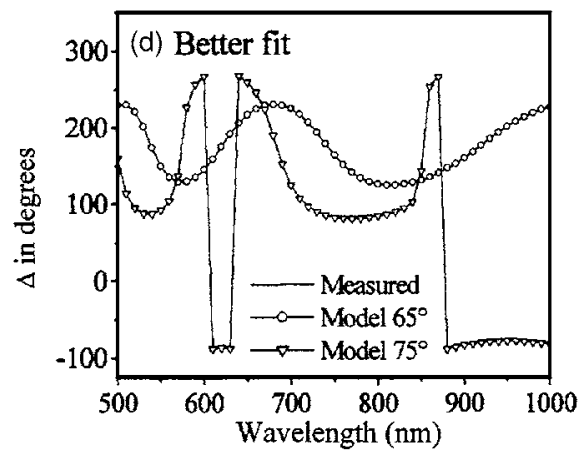

FIG. 5. Generated and experimental (a) and (b) using single BEMA model (MSE =77) and (c) and (d) using double BEMA model $(\mathrm{MSE}=3.5)$.
BEMA model $(\mathrm{MSE}=1)$. We then converted Cauchy and BEMA models to graded layers giving the same fit as nongraded fits $(\mathrm{MSE}=1)$. This suggested that the directly baked porous sample has a uniform refractive index throughout the sample and not graded.

For the bilayer porous samples, similar technique is performed in the analysis. The bilayer sample is first modeled as a single Cauchy layer and BEMA layer. As expected, the experimental and generated data do not fit very well given an MSE of 77 [see Figs. 5(a) and 5(b)]. The single layer model modified to graded model does not improved the fit very well $(\mathrm{MSE}=29)$. The model is then modified to two independent Cauchy layers and BEMA layers, improving the experimental and generated spectra significantly reducing the MSE fit to 3.5 [see Figs. 5(c) and 5(d)]. The ellipsometer results for bilayer sample are compared with Filmetric-F20 thin film measurement system. This technique is based on light reflectance. For the top film, our measurements show $199 \mathrm{~nm}$ thickness with a 1.197 refractive index, and for bottom film, $884.7 \mathrm{~nm}$ with 1.263 refractive index. This results agree with Filmetric measurement (top film: thickness $=228 \mathrm{~nm}, n$ $=1.21$; bottom film: thickness $=948 \mathrm{~nm}, n=1.27)$. The porosities of the bilayer film obtained from two BEMA models $(\mathrm{MSE}=3.5)$ were $48.1 \%$ for the top film and $31.8 \%$ for the bottom film. Figure 6 shows the depth profile of the graded bilayer films at $633 \mathrm{~nm}$ wavelength using two BEMA layers. The bilayer model is also modified to graded layer giving MSE of 3.1 (top film: thickness $=197.4 \mathrm{~nm}, n=1.195$, percent porosity $=48.6 \%$; bottom film: thickness $=886.2 \mathrm{~nm}, n$ $=1.262$, percent porosity $=31.8 \%$ ). The profile shows two distinct thicknesses and refractive index.

In the following discussion, we will show that a porous film can eventually be graded depending on different treatments the film undergoes. The closed-pore (OS7525) sample underwent Supercritical $\mathrm{CO}_{2}\left(\mathrm{SCCO}_{2}\right) /$ cosolvent process to remove the porogen, thus, making the film porous. The details of $\mathrm{SCCO}_{2}$ treatment are in Ref. 22. This processed film was then $\mathrm{O}_{2}$ plasma ashed $\left[\mathrm{CF}_{4} / \mathrm{O}_{2}(20 \mathrm{SCCM} / 80 \mathrm{SCCM})\right.$ for $1 \mathrm{~min}$ at $200 \mathrm{~W}$ plasma power]. It is suggested that $\mathrm{O}_{2}$ plasma ashing replaces- $\mathrm{CH}$ groups from the pore surface by substitution reaction causing the formation of polar $\mathrm{Si}-\mathrm{OH}$ in the following mechanism:

$$
\mathrm{Si}-\mathrm{CH}_{3}(s)+4 \mathrm{O}(g) \rightarrow \mathrm{Si}-\mathrm{OH}(s)+\mathrm{H}_{2} \mathrm{O}(g)+\mathrm{CO}_{2}(g) .
$$

The $\mathrm{OH}$-substituted endgroup is very favorable to attract moisture which in effect would increase the refractive index in time. ${ }^{24,31}$ Since treatment is at the surface of the film, CH groups would be reacted more at the topmost and closer regions where $\mathrm{O}$ diffusion accessibility is high. Consequently, $\mathrm{O}_{2}$ plasma damage (by $\mathrm{OH}$ substitution) is expected to be higher at the topmost layer of the film and decreases with respect to film depth, thus, a graded film is created.

For $\mathrm{O}_{2}$ plasma-ashed sample, we found that modeling it as a single layer gave a poor fit with MSE of 25. The model

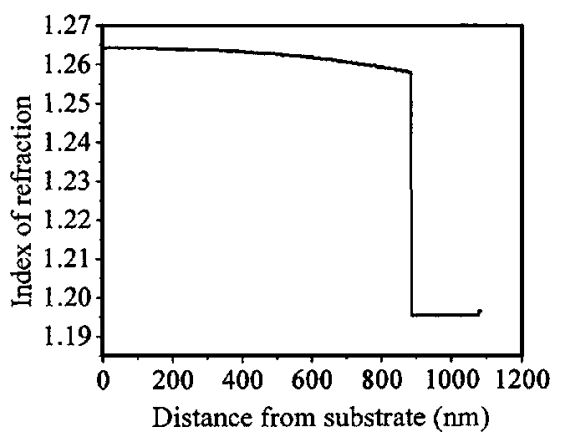

FIG. 6. Depth profile at $633 \mathrm{~nm}$ wavelength for bilayer porous film with different porogen loading. 


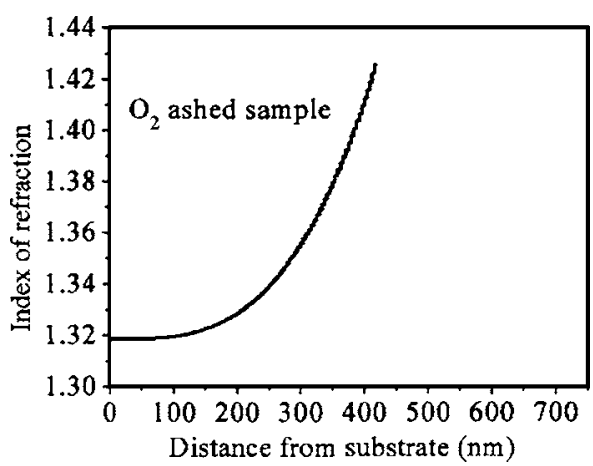

FIG. 7. Depth profile at $633 \mathrm{~nm}$ wavelength for $\mathrm{O}_{2}$ ashed.

is then modified to graded layer which improved the fit significantly with MSE of 4 . We have also tried modeling the sample as bilayer and graded bilayer, but it gave a poor fit with MSE of 30 and 20, respectively.

Figure 7 shows depth profile for $\mathrm{O}_{2}$ plasma-ashed film. We observed that this sample was indeed graded with a large variation in refractive index. We found that plasma damage occurs not only at the surface but into $3 / 4$ of the film thickness. The top surface of the ashed sample has higher refractive index, indicating the presence of $\mathrm{Si}-\mathrm{OH}$ bands. The above discussion shows the versatility of spectroscopic ellipsometry. Since the interlayer dielectric (ILD) films are exposed to $\mathrm{O}_{2}$ plasma and solvent cleaning to remove organic residual photoresist, it is important to understand the behavior of porous sample after the treatment. This ellipsometric analysis is crucial because it shows that oxygen plasma destroyed the film which may create a reliability problem.

\section{CONCLUSION}

Variable angle spectroscopic ellipsometer (VASETM) provides a practical means to characterize porous low- $k$ films. Spectroscopic ellipsometry cannot distinguish between open pores and closed pores, yet this method is a quick and effective tool to obtain refractive index depth profile, pore volume fraction aside from thickness and refractive index of single, and multilayer porous films. It is also a nondestructive measurement tool. For example, our VASE analysis has also shown that the interface of our porous film have higher porosity as compared with the bulk. In microelectronics industry, it is crucial to know the porosity distribution as a strategy to understand the behavior of porous sample. Increasing porosity drives the refractive index down, but degrades the mechanical stability and chemical properties of sample. Thus, detailed ellipsometer analysis for porous thin film will help with the next step of fabrication such as improving mechanical strength and pore sealing. In summary,

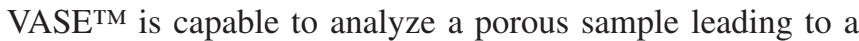
better understanding of its physical properties.

\section{ACKNOWLEDGMENTS}

The authors would like to acknowledge the financial support of Semiconductor Research Corporation (SRC).

${ }^{1}$ K. Maex, M. R. Baklanov, D. Shamiyan, F. Iacopi, S. H. Brongersma, and Z. S. Yanovitskaya, J. Appl. Phys. 93, 8793 (2003).

${ }^{2}$ D. Amans, S. Callards, A. Gagnaire, J. Joseph, G. Ledoux, and F. Huisken, J. Appl. Phys. 93, 4173 (2003).

${ }^{3}$ http://public.itrs.net. November 2004.

${ }^{4}$ C. J. Hawker, J. L. Hederick, R. D. Miller, and W. Volksen, U.S. Patent No. US 6,541,865 B2 (April 1, 2003).

${ }^{5}$ E. Kondoh, M. R. Baklanov, E. Lin, D. Gidley, and A. Nakashima, Jpn. J. Appl. Phys., Part 2 40, L323 (2001).

${ }^{6}$ S. Terada, T. Kinashi, and J. Spear, AIP Conf. Proc. 683, 546 (2003).

${ }^{7}$ J. Ilavsky, A. J. Allen, G. G. Long, and P. R. Jemian, Rev. Sci. Instrum. 73, 1660 (2002).

${ }^{8}$ A. A. Herrero, R. L. Heredero, E. Bernabeu, and D. Levy, Appl. Opt. 40, 527 (2001).

${ }^{9}$ M. R. Baklanov, K. P. Mogilnikov, V. G. Polovinkin, and F. N. Dultsev, J. Vac. Sci. Technol. B 18, 1385 (2000).

${ }^{10}$ K. P. Mogilnikov, V. G. Polovinkin, F. N. Dultsev, and M. R. Baklanov, Mater. Res. Soc. Symp. Proc. 565, 81 (1999).

${ }^{11}$ D. W. Gidley, W. E. Frieze, T. L. Dull, J. Sun, A. F. Yee, C. V. Nguyen, and D. Y. Yoon, Appl. Phys. Lett. 76, 1282 (2002).

${ }^{12} \mathrm{H}$. G. Tompkins and W. A. McGahan, Spectroscopic Ellipsometery and Reflectometry: A User's Guide (Wiley, New York, 1999).

${ }^{13}$ R. M. A. Azzam and N. M. Bashara, Ellipsometry and Polarized Light (North-Holland, Amsterdam, 1987).

${ }^{14}$ J. Woollam, B. Johs, C. Herzinger, J. Hilfiker, R. Synowicki, and C. Bungay, Critical Review of Optical Science and Technology-Optical Metrology, Proc. SPIE Vol. CR72, 3-28 (1999).

${ }^{15}$ D. Shamiryan, T. Abell, Q. T. Le, and K. Maex, Microelectron. Eng. 70, 341 (2003).

${ }^{16} \mathrm{~J}$. G. Webster, Wiley Encyclopedia of Electrical and Electronics Engineering Supplement (Wiley, New York, 2000), Vol. 1.

${ }^{17} \mathrm{Hect}$, Optics, 4th ed. (Addison-Wesley, Reading, 2002).

${ }^{18} \mathrm{M}$. Born and E. Wolf, Principles of Optics: Electromagnetic Theory of Propagation, Interference and Diffraction of Light, 7th ed. (Cambridge University Press, Cambridge 1999).

${ }^{19}$ F. L. Pedrotti and L. S. Pedrotti, Introduction to Optics, 2nd ed. (PrenticeHall, Englewood Cliffs, NJ, 1993).

${ }^{20}$ C. Himcinschi, M. Friedrich, C. Murray, I. Streiter, S. E. Schulz, T. Gessner, and D. R. T. Zahn, Semicond. Sci. Technol. 16, 806 (2001).

${ }^{21}$ H. C. Kim, J. B. Wilds, C. R. Kreller, W. Volksen, P. J. Brock, V. Y. Lee, T. Magbitang, J. L. Hedrick, C. J. Hawker, and R. D. Miller, Adv. Mater. (Weinheim, Ger.) 14, 1637 (2002).

${ }^{22}$ J. A. Lubguban et al., J. Mater. Res. 19, 3224 (2004).

${ }^{23}$ B. Lahlouh, J. A. Lubguban, G. Sivaraman, R. Gale, and S. Gangopadhyay, Electrochem. Solid-State Lett. 7, G338 (2004).

${ }^{24}$ C. M. Herzinger, B. Johs, W. A. McGahan, J. A. Woollam, and W. Paulson, J. Appl. Phys. 83, 3323 (1998).

${ }^{25}$ E. D. Palik, Handbook of Optical Constants of Solids (Academic, San Diego, 1998).

${ }^{26}$ A. V. Goncharenko, Phys. Rev. E 68, 041108 (2003).

${ }^{27}$ D. E. Aspnes, Thin Solid Films 89, 249 (1982).

${ }^{28}$ W. H. Press, B. P. Flannery, S. A. Teukolsky, and W. T. Vetterling, Numerical Recipes: The Art of Scientific Computing (Cambridge University Press, Cambridge, MA, 1988).

${ }^{29}$ B. Johs, J. A. Woollam, C. M. Herzinger, J. Hilfiker, R. Synowicki, and C. L. Bungay, Critical Reviews of Optical Science and Technology-Optical Metrology, Proc. SPIE Vol. CR72, 29-58 (1999).

${ }^{30}$ H.-C. Kim, W. Volksen, R. D. Miller, E. Huang, G. Yang, R. M. Briber, K. W. Shin, and S. K. Satija, Chem. Mater. 15, 609 (2003).

${ }^{31}$ P. T. Liu et al., Electrochem. Solid-State Lett. 5, G11 (2002). 